Schwendowius, Dorothee

\title{
Teilhabechancen in der Migrationsgesellschaft - Diskriminierungserfahrungen in der Schulbiographie
}

Haushalt in Bildung \& Forschung 5 (2016) 2, S. 19-34

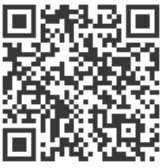

Quellenangabe/ Reference:

Schwendowius, Dorothee: Teilhabechancen in der Migrationsgesellschaft - Diskriminierungserfahrungen in der Schulbiographie - In: Haushalt in Bildung \& Forschung 5 (2016) 2, S. 19-34 - URN:

urn:nbn:de:0111-pedocs-203275 - DOI: 10.25656/01:20327

https://nbn-resolving.org/urn:nbn:de:0111-pedocs-203275

https://doi.org/10.25656/01:20327

in Kooperation mit / in cooperation with:

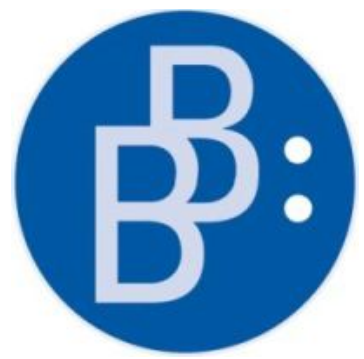

https://www.budrich.de

\section{Nutzungsbedingungen}

Gewährt wird ein nicht exklusives, nicht übertragbares, persönliches und beschränktes Recht auf Nutzung dieses Dokuments. Dieses Dokument ist ausschließlich für den persönlichen, nicht-kommerziellen Gebrauch bestimmt. Die Nutzung stellt keine Übertragung des Eigentumsrechts an diesem Dokument dar und gilt vorbehaltlich der folgenden Einschränkungen: Auf sämtlichen Kopien dieses Dokuments müssen alle Urheberrechtshinweise und sonstigen Hinweise auf gesetzlichen Schutz beibehalten werden. Sie dürfen dieses Dokument nicht in irgendeiner Weise abändern, noch dürfen Sie dieses Dokument für öffentliche oder kommerzielle Zwecke vervielfältigen, öffentlich ausstellen, aufführen, vertreiben oder anderweitig nutzen.

Mit der Verwendung dieses Dokuments erkennen Sie die Nutzungsbedingungen an.

\section{Terms of use}

We grant a non-exclusive, non-transferable, individual and limited right to using this document.

This document is solely intended for your personal, non-commercial use. Use of this document does not include any transfer of property rights and it is conditional to the following limitations: All of the copies of this documents mus retain all copyright information and other information regarding legal protection. You are not allowed to alter this document in any way, to copy it for public or commercial purposes, to exhibit the document in public, to perform, distribute or otherwise use the document in public.

By using this particular document, you accept the above-stated conditions of use.

\section{Kontakt / Contact:}

\section{peDOCS}

DIPF | Leibniz-Institut für Bildungsforschung und Bildungsinformation Informationszentrum (IZ) Bildung

E-Mail:pedocs@dipf.de

Internet: www.pedocs.de

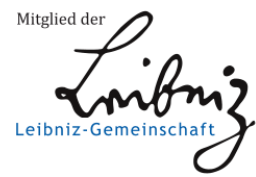


5. Jahrgang Heft 2

2016

궁

을

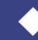

๘

잉

(ฮ)

는

过

정

은

힌

$\checkmark$

c

(1)

$\frac{0}{1}$

(

$\Phi$

$\xi$

$\frac{\pi}{\pi}$

$\stackrel{\infty}{乛}$

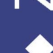

$\stackrel{ \pm}{ \pm}$

$\diamond$

$+$

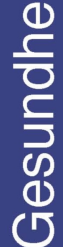

ISSN 2193-8806
Haushalt in

\section{Vielfalt erleben Vielfalt gestalten}




\section{Inhaltsverzeichnis}

Julia Kastrup \& Kirsten Schlegel-Matthies

Editorial.

Ansgar Stracke-Mertes

Der Index für Inklusion als Instrument der schulischen

Qualitätsentwicklung

Dorothee Schwendowius

Teilhabechancen in der Migrationsgesellschaft -

Diskriminierungserfahrungen in der Schulbiographie

Mona Massumi

Neu zugewanderte Kinder und Jugendliche in den Schulen - Potenziale

für die Ausbildung von Lehrkräften.

Barbara Fegebank

Vielfalt erleben - Vielfalt gestalten: Corporate Identity

der haushälterischen Bildung und der Haushaltswissenschaft

Karolin Wirth

Das Selbstlernzentrum als Instrument zur Implementation des Themas „Inklusion“ in die Lehrerbildung

Barbara Methfessel

Was sie begann, führte zum Erfolg!

Julia Kastrup

Rezension: Inklusion als Chance und Gewinn für

eine differenzierte Berufsbildung.

Julia Kastrup

Rezension: Haushaltsführung als Dienstleistung......

Marie Nölle

Rezension: Hauswirtschaftliche Betreuungsleistungen

personenorientiert durchführen 83 
Dorothee Schwendowius

\section{Teilhabechancen in der Migrationsgesellschaft - Diskriminierungserfahrungen in der Schulbiographie}

\section{Zusammenfassung}

Der Beitrag thematisiert die Frage, wie Teilhabe und Ausgrenzung im Schulsystem hergestellt und biographisch erfahren werden. Im Fokus steht die Rekonstruktion organisationaler und pädagogischer Praktiken, die zur Verfestigung migrationsgesellschaftlicher Macht- und Ungleichheitsverhältnisse beitragen. Auf Basis einer Studie zu Biographien und Bildungswegen von Lehramts- und Pädagogikstudierenden „mit Migrationsgeschichte“ werden Hindernisse der Teilhabe an schulischer Bildung aufgezeigt und Perspektiven für die Gestaltung von Schule und Lehrer(innen)bildung entworfen.

Schlüsselwörter: Schule, Migration, Diskriminierung, Biographie, Professionalisierung

\section{Einleitung}

Heterogenität im Bildungssystem ist im Rahmen der Debatte um Inklusion erneut stärker in den Fokus gerückt, wenngleich die erziehungswissenschaftliche Auseinandersetzung mit „Differenz“ eine weit längere Geschichte hat. Im schulpädagogischen Diskurs wird das Thema Differenz oft unter der Überschrift „Umgang mit Heterogenität" verhandelt (vgl. Budde, 2015). Mit dieser Formulierung wird meist die Frage verbunden, wie Schulen und Professionelle der Vielfalt der Lernenden angemessen begegnen können. Unter „Heterogenität“ wird indes Unterschiedliches verstanden - es geht u. a. um die Pluralität von Lernausgangslagen, um Leistungsdifferenzen, um sozio-ökonomische, kulturelle und Geschlechterdifferenzen.

Heterogenität erscheint in diesem Diskurs gleichsam als eine scheinbar natürliche Tatsache, die an bestimmte, vermeintlich objektive, „Merkmale“ der Lernenden geknüpft ist, auf die sich die Bildungseinrichtungen und Professionellen lediglich reaktiv beziehen. Der Anteil, den die Schule und die Professionellen an der Hervorbringung und Reproduktion solcher Differenzen und ihrer Umwandlung in (Bildungs-)Ungleichheiten haben, gerät dagegen oftmals aus dem Blick. In bildungssoziologischen Studien wird dagegen seit Langem thematisiert, dass das Bildungssystem nicht nur zum Abbau von Bildungsungleichheiten beiträgt, sondern im Gegenteil aktiv daran beteiligt ist, ungleiche Bildungsvoraussetzungen noch zu 


\section{Teilhabechancen in der Migrationsgesellschaft}

verschärfen (vgl. Bourdieu \& Passeron, 1971; Dravenau \& Groh-Samberg, 2005; Gomolla \& Radtke, 2009).

Der vorliegende Beitrag ${ }^{2}$ thematisiert die soziale Herstellung ungleicher Bildungs- und Teilhabechancen aus der Perspektive ehemaliger Schülerinnen. Die Grundlage dafür bilden die Ergebnisse einer biographisch-rekonstruktiven Studie zu Bildungswegen und Zugehörigkeitserfahrungen in der Migrationsgesellschaft (vgl. Schwendowius, 2015). In der Studie wurden die Lebensgeschichten von 15 Lehramts- und Pädagogikstudierenden, die selbst oder deren Eltern nach Deutschland migriert sind, aus biographie- und zugehörigkeitstheoretischer Perspektive untersucht. Im Folgenden wird zunächst die theoretisch-methodologische Perspektive skizziert, bevor anschließend anhand von Fallbeispielen aus den biographischen Erzählungen exemplarisch aufgezeigt wird, wie Teilhabe und Ausgrenzung im Bildungssystem von den Subjekten biographisch erfahren und „bearbeitet“ werden. Den Abschluss bilden einige Überlegungen zu möglichen Konsequenzen, die sich aus diesen Befunden für die Gestaltung von Schule und Lehrer(innen)bildung ziehen lassen.

\section{Biographieanalytische Perspektiven auf Teilhabe und Ausgrenzung im Bildungssystem}

Die öffentliche Diskussion um „Bildungserfolge“ und „-misserfolge“ in der Migrationsgesellschaft wird meist durch die Ergebnisse von large-scale-Analysen dominiert, die auf statistische Zusammenhänge zwischen Schulerfolg, sozialer Herkunft und dem „Migrationshintergrund“ von Schülerinnen und Schülern aufmerksam machen (z. B. Baumert, Stanat, Watermann, 2006). Dies allein trägt indes wenig zur Aufklärung der konkreten (Re-)Produktionsprozesse von Ungleichheiten im Bildungssystem bei. Einen anderen Zugang wählen interpretative Studien, die an der Rekonstruktion der sozialen Prozesse und Praktiken ansetzen, in denen Einund Ausschlüsse im Bildungssystem erzeugt (vgl. z. B. Bräu \& Schlickum, 2015) und biographisch erfahren werden. Biographieanalytische Forschungsansätze stehen in diesem Zusammenhang für einen Zugang, der es ermöglicht, Ein- und Ausschlusserfahrungen im Bildungssystem aus der Binnensicht der Subjekte zu rekonstruieren (vgl. Dausien, Rothe, Schwendowius, i. E.).

Das spezifische Potenzial eines biographieanalytischen Forschungszugangs für die Untersuchung von Bildungswegen liegt darin, Ein- und Ausschlussprozesse und -erfahrungen im lebensgeschichtlichen Prozessverlauf und im Zusammenspiel institutioneller und lebensweltlicher Bedingungen untersuchen zu können. Biographieanalysen ermöglichen dabei einerseits Einsichten in Bildungsverläufe in ihrer äußeren Gestalt. Sie geben Aufschluss über Ressourcen und Gelegenheitsstrukturen, die Teilhabe an höherer Formalbildung ermöglichen, ebenso wie über Bedingungen, die diese Teilhabe erschweren. Andererseits lassen sich Erfahrungen mit 


\section{Teilhabechancen in der Migrationsgesellschaft |}

Zugehörigkeit und Ausgrenzung sowie ihre biographische Bearbeitung und Bedeutung für die Zugehörigkeitsverständnisse der Subjekte herausarbeiten.

Lebensgeschichtliche Erzählungen sind dabei als historisch und kulturell spezifische Formen der Selbstpräsentation zu verstehen (vgl. Hahn, 2000). Sie bilden das Erlebte nicht spiegelbildlich ab, sondern stellen vielmehr retrospektive Vergegenwärtigungen der Vergangenheit dar, die vor dem Hintergrund der jeweils antizipierten Zukunft vorgenommen werden (vgl. v. Engelhardt, 2011; Rosenthal, 2005). Biographien verweisen auf Prozesse individualgeschichtlicher „Erfahrungsaufschichtung" (Schütze, 1987), zugleich sind sie aber immer auch Dokumente des Sozialen insofern als in ihnen überindividuelle Erfahrungen und historischgesellschaftliche Strukturvorgaben, Möglichkeitsräume und Grenzen sichtbar werden, welche die Lebensgeschichten und Selbstentwürfe der Subjekte strukturieren (vgl. Dausien, 1996). Dazu gehören auch gesellschaftliche Zugehörigkeits- und Differenzordnungen (Mecheril \& Plößer, 2011), die sich in sozialen und institutionellen Kontexten, die die Subjekte im lebensgeschichtlichen Verlauf „durchqueren", in unterschiedlicher Weise manifestieren und konkretisieren. Bildungskontexte lassen sich in diesem Sinn als "Zugehörigkeitskontexte“ (Mecheril, 2003, S. 128) verstehen, die den Subjekten unterschiedliche Selbstverortungen ermöglichen (oder verunmöglichen). Biographieanalytisch betrachtet lassen sie sich auch als Räume verstehen, in denen sich Selbst- und Zugehörigkeitsverständnisse der Subjekte prozesshaft formieren und transformieren.

Die folgenden Ausführungen konzentrieren sich auf die Rekonstruktion von biographischen Erfahrungen im Schulsystem. Es geht um die konkreten institutionellen Kontexte und pädagogischen Praktiken der schulischen (Un-) Gleichbehandlung, die eine gleichberechtigte Bildungsteilhabe von Schülerinnen und Schülern, die über Migrationserfahrungen verfügen oder als „Migrationsandere“ (vgl. Mecheril, 2004) klassifiziert werden, behindern. Ausgehend von den biographischen Erzählungen von Studierenden wird exemplarisch gezeigt, welche Bedingungen die Teilhabe an (höherer) Bildung erschweren bzw. dazu beitragen, dass die Biographinnen und Biographen sich in schulischen Bildungsräumen nicht als ,fraglos zugehörig" (Mecheril, 2003, S. 144) präsentieren können. Die Egebnisse der Rekonstruktionen werden in zwei (nicht immer ganz trennscharfe) Dimensionen getrennt: Zunächst werden Erfahrungen mit Barrieren an schulischen Übergängen in den Blick genommen, bevor anschließend Diskriminierungserfahrungen im unterrichtlichen und schulorganisatorischen Kontext thematisiert werden. 


\section{Diskriminierende Zuweisungspraktiken an Schulübergängen}

Die Bedeutung von schulischen Übergängen für die Verteilung von Bildungschancen im deutschen Schulsystem sind ebenso bekannt wie der Umstand, dass Schülerinnen „mit Migrationsgeschichte“ oft in besonderem Maße negativ davon betroffen sind. In biographieanalytischer Perspektive wird indes deutlicher sichtbar, welche Bedingungskonstellationen Schulübergänge strukturieren, welche Erfahrungen sich damit für die Subjekte verbinden und welche Konsequenzen sie für die Schulbiographien haben.

Die Bedeutung von diskriminierenden Logiken und Praktiken an Schulübergängen wird im untersuchten Sample insbesondere in den Erzählungen so genannter "Quereinsteiger“ deutlich, d. h., von Schülerinnen und Schülern, die vor ihrer Migration nach Deutschland die Schule in ihrem jeweiligen Herkunftsland besucht haben. Ihre Erzählungen zeigen, dass Kinder, die nicht dem Ideal der einsprachigen, im deutschen Schulsystem und der standarddeutschen Sprache sozialisierten Schülerin entsprechen, oftmals fraglos in Hauptschullaufbahnen einmünden - unabhängig von ihren bisherigen Schulleistungen. Ein Hintergrund dafür ist der Umstand, dass die Hauptschule (insbesondere in ländlichen Regionen) in der Schulzeit der interviewten Studierenden meist die einzige weiterführende Schulform war, die über eine Möglichkeit der Deutschförderung verfügte. ${ }^{2}$ Um den Kindern diese Förderung zu ermöglichen, erscheint der Übertritt an die Hauptschule den beteiligten Akteuren daher oft als alternativlos; andere Schulformen geraten dagegen gar nicht erst in den Blick. Die Schülerinnen und Schüler werden so nach ihrem Eintritt ins deutsche Bildungssystem in eine schulische Bahn gelenkt, die nicht auf höhere Bildung ausgelegt ist - obwohl sie und oft auch ihre Familien höhere Bildungsaspirationen haben. Dieses Schulverlaufsmuster verweist auf eine Form indirekter institutioneller Diskriminierung (Gomolla \& Radtke, 2009, S. 48ff.) von Kindern und Jugendlichen mit einem (zugeschriebenen) Förderbedarf in der standarddeutschen Sprache.

Wie sich in den Erzählungen zeigt, hat die unterschiedslose Zuweisung zur Hauptschule auch weitreichende Folgen für die weitere Schulbiographie, denn die Schülerinnen müssen sich ihren schulischen Aufstieg im Widerstand gegen das ihnen institutionell nahegelegte Hauptschullaufbahnmuster erkämpfen, das ihre Zukunft bereits vorzuzeichnen scheint. Aufstiegsorientierte Schülerinnen und Schüler müssen dabei den erlebten Widerspruch zwischen dem meritokratischen Versprechen, dass das Schulsystem nach dem Leistungsprinzip funktioniere und nach oben durchlässig sei, und konträren eigenen Erfahrungen individuellbiographisch bewältigen. Dies soll an einem Beispiel verdeutlicht werden.

Die interviewte Studentin, die hier „Anna Schuster“ genannt wird, kam im A1ter von zehn Jahren mit ihrer Familie aus Russland in eine deutsche Kleinstadt im 


\section{Teilhabechancen in der Migrationsgesellschaft |}

ländlichen Raum. Vor dem Hintergrund der Unterversorgung mit Deutschfördermöglichkeiten tritt sie in die örtliche Hauptschule über, die als einzige Schulform ein solches Angebot vorhält. Vor dem Hintergrund von Erfahrungen mit staatlich verwehrten Bildungs- und Berufsmöglichkeiten in der Elterngeneration und orientiert am gesellschaftlichen „Wert“ von Bildungsabschlüssen verfolgt Anna Schuster jedoch den Entwurf, einen höherwertigen Schulabschluss zu erlangen. Nachdem ihr die Fortsetzung ihrer Schullaufbahn zunächst verwehrt bleibt - der Hintergrund ist eine schlechte Benotung in der Englischprüfung, die aber nach einer weiteren Prüfung bei einer anderen Lehrkraft aufgehoben wird - erlangt die Biographin schließlich den erweiterten Hauptschulabschluss mit Qualifikation für die gymnasiale Oberstufe. Über ihre Erfahrungen nach dem Wechsel in die elfte Klasse des örtlichen Gymnasiums erzählt sie Folgendes:
A: also für mich wars eben auch - ne große Leistung dass ich die Qualifikation be- kommen hatte trotz dem dass mein Klassenlehrer mich für doof hielt. Und dann dieser Rückschlag in der Oberstufe - (wir) hatten halt $\mathrm{n}$ Deutschlehrer der uns eben direkt auch an den Kopf geworfen hatte und gesagt also ein Hauptschüler hat bei uns am Gymnasium noch nie ein Abitur bestanden. So. Und das hat er uns JEDE Deutschstunde gesagt und ihr schafft das sowieso nicht auch wenn ihr bis zur Zwölf bleibt ihr werdet abgehen ihr schafft das nicht ihr werdet nie das Stadium er- reichen was die Gymnasiasten haben - also das war wirklich hart also jemand der einen wirklich rausgeekelt hat. Und das vor der ganzen Klasse. (17/35-18/10)

Das Versprechen, dass ein Aufstieg durch Leistung möglich ist, wird hier offen gebrochen. Nach der mühevoll erlangten Qualifikation konfrontiert der Lehrer die ehemaligen Hauptschüler mit einer kollektiven Negativprognose für ihre weitere Schullaufbahn. Ihre schulische Vorgeschichte wird zu einem Stigma, das den weiteren Schulverlauf zu determinieren scheint. Für Anna Schuster bedeutet dies eine Missachtung des für sie zentralen Erfolgs, den Qualifikationsvermerk für die Oberstufe erhalten zu haben. Vor allem aber erweist sich ihr schulisches Handlungsmuster, durch das Erbringen guter schulischer Leistungen auf einen ,vernünftigen Abschluss" hinzuarbeiten, als wirkungslos.

Angesichts dieser Erfahrung, die die Biographin hier zum wiederholten Male macht, zieht sie in Erwägung, das Gymnasium zu verlassen und stattdessen eine Ausbildung zu beginnen. Zwar gelingt es ihr - unterstützt durch die Ermutigung ihrer Eltern - am Ende doch, über den Umweg einer Klassenwiederholung das Abitur zu machen. Aber sie konstruiert sich in weiten Teilen ihrer Erzählung nicht als Akteurin ihrer Schulbiographie, sondern als jemand, die immer wieder Entscheidungen und Vorgängen ausgeliefert ist, auf die sie selbst keinen Einfluss hat.

Aber auch in den Erzählungen von Studierenden, die von Beginn an in Deutschland die Schule besucht haben, wird die Bedeutung diskriminierender Zuweisungspraktiken an Schulübergängen deutlich. Dazu ein zweites Beispiel: Die Interviewte, „Bahar Merizadi““, ist als Kleinkind mit ihrer Familie aus einer irani- 


\title{
Teilhabechancen in der Migrationsgesellschaft
}

schen in eine deutsche Großstadt migriert. Sie besucht dort eine integrierte Grundund Gesamtschule. Nachdem sie die zehnte Klasse mit Auszeichnung beendet hat, verfolgt sie zunächst den Plan einer künstlerisch orientierten Ausbildung, bevor sie beschließt, doch das Abitur machen zu wollen. Über die Anmeldung am Gymnasium und anschließenden Besuch der Oberstufe berichtet sie Folgendes:

\begin{abstract}
B: und das Lustige bei der Schule war, das ist ja halt in diesem sozialen Brennpunkt, und in der Klasse ist dann halt irgendwie rausgekommen, weil mich jemand gefragt hat, hast du dich direkt hier beworben? Meint ich nein, ich hab mich in ner anderen Schule beworben, das - wär halt auch n guter Stadtteil gewesen, aber es hieß es ist zu voll und deswegen wurd ich hier rübergeschickt. Und dann musste dir vorstellen von 25 Schülern haben 20 Schüler genau dasselbe erzählt. Also und das waren alles Ausländer. Von Russen Polen Afghanen Iranern Türken - alle wurden sie hierher geschickt weil es hieß die hätten keinen Platz mehr. (Bahar Merizadi $6 / 31-39$ )
\end{abstract}

Die Erzählung der Interviewten legt eine Deutung des Geschehens als eine direkte Form von institutionellem Rassismus nahe: Die Schülerinnen und Schüler werden (vermutlich aufgrund ihres Namens) nach ihren Herkunftshintergründen unterschieden und in verschiedene Schulen kanalisiert. Diese unterscheiden sich nicht nur hinsichtlich ihres Prestiges und der sozialen Zusammensetzung der Schülerschaft erheblich voneinander. Auch hinsichtlich des fachlichen Angebotsspektrums und der Wahlmöglichkeiten bestehen Differenzen. Daraus ergeben sich, wie Bahar Merizadis Erzählung zeigt, weitreichende Folgen für die Schullaufbahnen der Schülerinnen und Schüler.

B: Englisch mussten wir - auf eine andere Schule, B-Schule, gehen, weil wir bei uns - kam kein Leistungskurs zustande. - Weil die waren einfach alle - /(lachend) zu schlecht/ fürs Englisch und - wir mussten dann immer nach Stadtteil P. Stadtteil $P$ ist auch so ne Gegend die ist echt $-\mathrm{ja}$, auch schon gut angesehen. (...) Und da waren ja auch Ausländer - auf der B-Schule aber nicht so viele, vielleicht ne Handvoll - aber allein schon dass es hieß so okay, da kommen die Reuterplatzleute - Leute - die jetzt hier in den LK kommen - und wir kamen da an, ph, haben uns gefreut und dachten so, cool, hi, okay, ich bin die und die - die haben kein Wort mit mir geredet. (1) Mit uns allen nicht. (...) Und das haben wir schon sehr gemerkt, also dass die einfach uns sehr ausgegrenzt haben (...) und letzten Endes waren wir von fünf Leuten, die den Leistungskurs Englisch gemacht haben, bin ich als Einzige da geblieben, weil die gesagt haben, ich kann das nicht - nervlich und von den Leistungen die da erwartet werden - ich war die Letzte die da geblieben ist, und ich hab mit Fleiß und allem hab ich das da auch geschafft. (Bahar Merizadi 40/15-40)

Es zeigt sich hier ein Phänomen, das in ähnlicher Form auch noch in anderen Interviews sichtbar wird: Die Schülerinnen und Schüler müssen für einen Leistungskurs in eine andere Schule wechseln, die im Gegensatz zu ihrer Schule in einem ,gut angesehen[en] Stadtteil“" liegt. Dies ist nicht nur mit einem organisatorischen 


\section{Teilhabechancen in der Migrationsgesellschaft}

Mehraufwand für die Einzelnen verbunden, sondern es haftet den Schülerinnen und Schülern das Stigma (vgl. Goffman, 1974) einer Schule im „sozialen Brennpunkt“ an, gegen das sie individuell und kollektiv ankämpfen müssen. Die Belastungen, die mit dieser Stigmatisierung und mit den unterschiedlichen Leistungserwartungen an den zwei parallel besuchten Schulen verbunden sind, haben zur Folge, dass fast alle Schülerinnen und Schüler, die dies betrifft, ihre Abiturambitionen aufgeben. Dies kann in den Worten von Pierre Bourdieu und Jean-Claude Passeron (2007 [1964], S. 18) als ein Mechanismus der Abdrängung gedeutet werden: Die erschwerenden Bedingungen führen zu einem Selbstausschluss der betroffenen Jugendlichen aus den sozial abgewerteten Stadtteilen - zugleich sind dies Schülerinnen und Schüler „mit Migrationsgeschichte“ - aus der gymnasialen Laufbahn.

Indem die Interviewte sich zuschreibt, dem Ausschließungsprozess durch „Fleiß und alles“ aktiv entgegengearbeitet zu haben, deutet sie ihren Schulerfolg als Folge besonderer, individueller Anstrengungen. Darüber hinaus gibt es in ihrer Erzählung immer wieder Hinweise darauf, dass es ihr - trotz der erfahrenen rassistischen Diskriminierung - gelingt, an einer distinktiven Selbstkonstruktion als Schülerin ,,aus gutem Haus“ festzuhalten und dies zu einer biographischen Ressource (vgl. Hoerning, 1989) zu machen, die ihr das Festhalten an ihrem bildungsbiographischen Entwurf ermöglicht.

Die Beispiele verdeutlichen die nachhaltige Relevanz diskriminierender $\mathrm{Zu}$ weisungspraktiken an Schulübergängen für die Bildungsbiographien der Subjekte. Dabei wird deutlich, dass Diskriminierungserfahrungen an Schulübergängen oft in einem breiteren Kontext schulorganisatorischer Prozesse stehen, in denen von $\mathrm{Zu}$ schreibungen sprachlicher bzw. „ethnischer“ Differenz Gebrauch gemacht wird, die für die Biographinnen zu Hindernissen auf dem Weg zu (höherer) Bildung werden.

\section{Diskriminierung im schulorganisatorischen und unterrichtlichen Setting}

Nicht nur an Bildungsübergängen, sondern auch im Kontext der Unterrichtsorganisation und -praxis sind organisationale Strukturen und pädagogische Routinen daran beteiligt, Ausschlüsse herzustellen, Zugehörigkeitserfahrungen zu erschweren und migrationsgesellschaftliche Macht- und Differenzordnungen $\mathrm{zu}$ bestätigen. Dazu tragen erstens eine institutionelle Logik des Ignorierens der heterogenen Bildungsbiographien und eine fehlende Anerkennung der Ressourcen der Lernenden bei. Zweitens verweisen die Erzählungen auf Praktiken der Ethnisierung bzw. des Othering in der Unterrichtsinteraktion und -organisation, durch die die Subjekte als Migrationsandere positioniert werden. 


\section{Teilhabechancen in der Migrationsgesellschaft}

\subsection{Ignorieren von „Differenz“}

Die Bedeutung von „Differenzunempfindlichkeit“ und des Ignorierens schulisch relevanter Sozialisationserfahrungen lässt sich erneut an der Geschichte von „Anna Schuster" aufzeigen. In der folgenden Passage berichtet sie über ihre Unterrichtserfahrungen, die sie nach ihrem Übertritt an die Hauptschule unmittelbar nach der Migration aus Russland macht:

A: das war für mich eine neue Welt dann die Schule an sich - im Schulalltag im Unterricht diese Freiheit dass man die Füße aufn Tisch legt dass man den Lehrer einfach mal zurück sagt dass man einfach im Klassenraum schreit also es war für mich alles komplett neu, ich war wirklich Disziplin und Ordnung gewöhnt und dass man wirklich strammsaß und aufzeigte und aufstand und auswendig lernte und nach vorne an die Tafel ging, das war ja alles nicht gegeben also [...] ich war eher dieses Disziplinierte gewöhnt und hab mich da auch - wohler gefühlt wenn es auch hier an der Hauptschule einen Lehrer gab der mehr auf Disziplin geachtet hat weil dann hab ich persönlich einfach mehr lernen können als in diesem lauten Umfeld. $(2 / 26-3 / 7)$

Das Unterrichtsmilieu der Hauptschule, in die Anna Schuster nach der Migration nach Deutschland übertritt, ist kein Kontext, in dem sie sich einbringen und sich als wirksam (vgl. Mecheril, 2003, S. 169ff.) erfahren kann. Die Voraussetzung dafür wäre hier u. a. die Fähigkeit, sich durch unübersichtliche Situationen nicht irritieren $\mathrm{zu}$ lassen und die eigene Beteiligung am Unterricht notfalls lautstark zu erkämpfen. Diese Voraussetzungen sind nicht Teil von Anna Schusters schulischem Handlungsrepertoire. Sie verfügt über andere Muster des „doing student“ (vgl. Faulstich-Wieland, Weber, Willems, 2004) aus einem Schulsystem, in dem Disziplin und Konformität gefordert waren, um schulischen Erfolg haben zu können.

Die Ausschlusserfahrungen der Biographin sind aber nicht nur Folge der fehlenden Passung zwischen einem bestimmten Schülerinnenhabitus und der Kultur der betreffenden Hauptschule (vgl. Kramer \& Helsper, 2011). Sie verweisen auch auf schulorganisatorische Strukturen und Praktiken, die Ressourcen und Unterstützungsbedarfe von mehrsprachigen Schülern und Schülerinnen aus anderen nationalen Schulsystemen ignorieren: Anna Schuster verfügt beim Eintritt ins deutsche Schulsystem noch nicht über die (in der Schule einzig legitime) standarddeutsche Sprache. Ihre mitgebrachte Schulsprache (Russisch) und ihre familiensprachlichen Kompetenzen (ein niederdeutscher Dialekt) erfahren keine Anerkennung oder Förderung. Es besteht z. B. keine Möglichkeit, Russisch als erste Fremdsprache weiterzuführen. Stattdessen muss Anna Schuster wie alle anderen Schüler zeitgleich neben Deutsch auch Englisch erlernen, was sie als extrem belastend erlebt. Beim Erlernen der lateinischen Schrift erhält sie wenig Unterstützung und wird durch ihren Lehrer für ihre Handschrift extrem abgewertet. Auch erwähnt sie an keiner Stelle, dass Lehrkräfte einschreiten würden, wenn sie von Mitschülerinnen aufgrund ihres Dialekts ausgelacht wird. 


\section{Teilhabechancen in der Migrationsgesellschaft}

Dass der Heterogenität schulischer Vorerfahrungen und sprachlicher Voraussetzungen der Schülerinnen und Schüler oft durch eine Praxis der Ignoranz und der Assimilation begegnet wird (vgl. Wenning, 2007, S. 27) zeigt sich auch in anderen Interviews. Außer separaten Deutschförderklassen, die von den Interviewten als ausgrenzend empfunden werden, kommen keinerlei institutionalisierte Antworten auf die Anwesenheit von so genannten „Seiteneinsteigern“ zur Sprache. Dies ist insofern bemerkenswert als dies die Zeit Anfang der 1990er Jahre betrifft, in der die Zahl von ausgesiedelten Schülern bundesweit den Höhepunkt erreichte (vgl. Bade \& Oltmer, 1999). Für die Subjekte sind mit dem Übergang aus anderen nationalen Bildungssystemen deshalb oft sprachliche, leistungsbezogene und kulturelle Entwertungsprozesse verbunden, die sich auch in den biographischen Konstruktionen, Habitus und Selbstdeutungen niederschlagen.

Diese Ausschlusserfahrungen lassen sich auch als Ausdruck fehlender Strukturen und Kulturen der Anerkennung von „Differenz“ innerhalb der Organisation Schule verstehen. Sie resultieren aus den Funktionsweisen eines Schulsystems, das auf der unhinterfragten Normalitätskonstruktion von monolingual (vgl. Gogolin, 2008) und mononational verfassten Schülerbiographien basiert. Diese Normalitätserwartung wird in verschiedenen schulischen Milieus und Kollegien in unterschiedlichem Maße reflektiert. Anna Schusters Erzählung deutet darauf hin, dass es in der von ihr besuchten Schule keine Reflexionskultur für die Ausschließungen gibt, die durch die unhinterfragte Normalitätskonstruktion produziert werden. Stattdessen scheinen wahrgenommene Probleme mit „Differenz“ im Kollegium bearbeitet $\mathrm{zu}$ werden, indem sie den ausgesiedelten Schülerinnen angelastet werden, die dabei als eine homogene Problemgruppe erscheinen.

\subsection{Ethnisierung und Othering}

Das Ignorieren der heterogenen sozio-kulturellen und sprachlichen Hintergründe der Lernenden ist jedoch nur eine Variante des schulischen Umgangs mit „Differenz". Kontrastierend dazu ließen sich Praktiken ethnisierender Unterscheidung rekonstruieren, durch die (zugeschriebene) ethnisch-kulturelle Differenzen dramatisiert werden. Die Schülerinnen und Schüler lernen dadurch, sich als „Andere“ zu verstehen. In einigen Erzählungen wird die Kategorie „Migration“ durch solche Differenzierungspraktiken überhaupt erst zu einer bedeutsamen Dimension der biographischen Selbstbeschreibung.

Ein Beispiel dafür ist die Erzählung von „Meral Yilmaz“, deren Eltern in den 1960er Jahren im Zuge des Anwerbeankommens mit der Türkei nach Deutschland kamen. Meral Yilmaz wächst in einer deutschen Kleinstadt auf und besucht das dortige Gymnasium. Als eine der wenigen Schülerinnen, die hier ein Kopftuch tragen, wird sie von ihren Mitschülerinnen und -schülern ebenso wie von den Lehrkräften als „Muslima“ identifiziert. Obwohl sie im Interview bemüht ist, Dis- 


\section{Teilhabechancen in der Migrationsgesellschaft}

kriminierungserfahrungen zu relativieren, beschreibt sie sich als der Schule nicht sozial zugehörig. Sie berichtet z. B. Folgendes:

M: sobald das Thema Islam in der Schule aufkam, wurden wir sozusagen als muslimische Schüler /(lachend) beauftragt/ was zu erzählen. (...) Und - ich kann mich erinnern, sobald ein Thema über Islam dann - in der Klasse aufkam, wollte ich zwar etwas sagen, aber ich hatte Bedenken, dass ich das nicht richtig erklären kann und aufgrund dessen /(lachend) raste mein Herz so stark/! So ja, da war ich eher so zurückhaltend /(lachend) und hab nichts gesagt/. (...) Also es betrifft mich persönlich, es ist für mich sehr wichtig, aber ich weiß auch die Vorurteile der anderen, was die über - meine Religion vielleicht denken, und man hat Angst diese Vorurteile zu bekräftigen indem man vielleicht etwas Falsches sagt. Ne, und das war schon - an solchen Situationen fand ich das nicht so schön, also es war sehr bedrückend. (Meral Yilmaz 30/23-31/9)

Meral Yilmaz kritisiert das Interesse der Lehrkräfte an den kulturellen Zugehörigkeiten der Schülerinnen und Schüler als sehr partiell. Es reduziert sich auf bestimmte Anlässe, bei denen ihnen unterstellt wird, dass sie als „Expertinnen“ allgemeingültige Auskünfte über eine „fremde Kultur“ oder eine Religion geben können. Meral Yilmaz beschreibt hier eine Praktik, die in den Postcolonial Studies als Othering („Ver-Anderung“) bezeichnet wird (vgl. Said, 1978). Bei der Aufforderung, etwas zum Islam zu erzählen, geht es nicht darum, dass die Schülerin über ihre persönliche religiöse Auffassung und Praxis berichten soll, sondern sie soll für den Islam im Allgemeinen sprechen. Meral Yilmaz wird also als Repräsentantin „des“ Islam adressiert und dadurch in die Position der „Religionsanderen“ verwiesen (Mecheril \& Olalde, 2011).

Die Aufforderung, etwas zum ,Thema Islam“ zu sagen ist dabei durchaus ambivalent - dies zeigt sich darin, dass die Biographin sich nicht nur als passive Adressatin positioniert, sondern sich die Aufforderung auch zu Eigen macht: Sie „wollte“ etwas sagen. Dabei ergibt sich allerdings ein Dilemma: Nimmt sie den Auftrag an, so trägt sie eine hohe Verantwortung dafür, komplexe religiöse $\mathrm{Zu}$ sammenhänge auch „richtig“ zu „erklären“. Gelingt ihr dies nicht, so riskiert sie, Vorurteile gegen „den“ Islam zu bestätigen und wird selbst als (gläubige) Person diskreditierbar. Auch in einer weiteren Episode wird ihre religiöse Zugehörigkeit zum Anlass für Diskriminierung:

M: und ja - was ich nie vergessen werde, ist dass ich in der Schule in der Oberstufe in der elften Klasse, ich hatte katholische Religion gewählt, und im ersten Halbjahr durfte ich dran teilnehmen und im zweiten Halbjahr kam ein anderer Lehrer, und dieser Lehrer hat mich in der ersten Stunde - vor den ganzen Schülern hat er mich rausgeholt - und mich draußen gebeten, dass ich zum Sekretariat gehen solle und mich zum Philosophiekurs anmelden solle weil er nicht möchte dass ein muslimisches Kind in seinem Religionsunterricht teilnimmt. Das war so das Schlimmste was ich in meiner Schullaufbahn so erlebt hab so - als - Diskriminierung so zu sagen. Wo ich gedacht habe, was ist denn jetzt los. (Meral Yilmaz 6/36-7/6) 


\section{Teilhabechancen in der Migrationsgesellschaft}

Durch ihre Teilnahme am katholischen Religionsunterricht irritiert Meral Yilmaz offenbar die Grenzen der dominanten Zugehörigkeitsordnung (vgl. Mecheril, 2003). Durch den Akt des öffentlichen Ausschließens der muslimischen Schülerin wird diese Ordnung durch den Lehrer symbolisch wiederhergestellt. In diesem Prozess werden die Schülerinnen und Schüler entlang ihrer religiösen Zugehörigkeit als Mitglieder oder Nichtmitglieder identifiziert und bestätigt.

In der biographischen Erzählung von Meral Yilmaz werden diese Erfahrungen bearbeitet, indem sie ihre soziale Welt als zweigeteilt konstruiert - auf der einen Seite stehen ihre Familie und das muslimische Jugendzentrum, in denen Zugehörigkeitserfahrungen möglich werden und in denen sie sich selbst als aktives Subjekt konstruiert, auf der anderen Seite steht die Schule, die sie zwar formal erfolgreich durchläuft, zu der sie sich aber nicht als zugehörig präsentieren kann.

\section{Perspektiven für die Gestaltung von Schule und Lehrer(innen)bildung}

In den biographischen Erzählungen lassen sich - durch die Perspektive der Subjekte ,hindurch“" diskriminierende Strukturen und Praktiken im Schulsystem rekonstruieren, die zur Herstellung ungleicher Teilhabechancen beitragen und hierarchische Zugehörigkeitsordnungen bestätigen. Schulische Diskriminierung nimmt dabei unterschiedliche Formen an. Nicht immer sind die Logiken der Behinderung von Teilhabe und Zugehörigkeit auf den ersten Blick offen erkennbar. Vielmehr ist Diskriminierung oftmals eine Begegnung mit „unsichtbare[n], unausgesprochene[n] und unbemerkte[n] Hindernisse[n]“ (Terkessidis, 2010, S. 9), die in den Bildungswegen der Subjekte kumulieren.

Für die Herstellung von Ein- und Ausschlüssen in der Schulbiographie spielen Lehrpersonen in den untersuchten Erzählungen eine wichtige Rolle. Sie nehmen die Rolle von Gatekeepern (vgl. Behrens \& Rabe-Kleberg, 1992) ein, die an schulischen Übergängen durch ihre Entscheidungen oder Empfehlungen den weiteren Verlauf des Bildungswegs maßgeblich mit strukturieren. Sie können biographische Optionen eröffnen oder Zugänge verschließen, Bildungswege unterstützen oder behindern. In vielen Erzählungen treten sie zudem auch als Orientierungspersonen und Vorbilder in Erscheinung.

Dennoch würde es zu kurz greifen, den Blick einzig auf die „Einstellungen“ und das Handeln einzelner Lehrkräfte zu richten, wenn es um die Vermeidung von Diskriminierung geht. In den Rekonstruktionen wird vielmehr erkennbar, dass den Ausschlussmechanismen nicht in jedem Fall negative Absichten oder Vorurteile einzelner Lehrkräfte zugrunde liegen. Sie verweisen vielmehr darauf, dass Diskriminierung auch im breiteren Kontext schul- und unterrichtsorganisatorischer Handlungslogiken und -routinen sowie kollegial geteilter Normalitätserwartungen und 


\section{Teilhabechancen in der Migrationsgesellschaft}

professioneller Selbstverständnisse verstanden und untersucht werden muss. Die Strukturen der jeweiligen Einzelschule, die organisationalen und unterrichtlichen Kulturen des (praktischen und reflexiven) „Umgangs mit Differenz“ bilden je spezifische Kontexte für den Entwurf professioneller Selbstverständnisse und die Entwicklung und Legitimation von Handlungsroutinen. Bemühungen um mehr Teilhabegerechtigkeit im Bildungswesen der Migrationsgesellschaft müssen folglich konsequent die Schule als Gesamtorganisation in den Blick nehmen. Für die Programmatik einer diskriminierungskritischen Entwicklung auf der Ebene von Einzelschulen (vgl. Karakaşoğlu, Gruhn \& Wojcziechowicz, 2011) bedeutet dies, dass die oftmals ganz unhinterfragt bleibenden institutionellen und pädagogischen Routinen, Normalitätserwartungen und kollegial geteilten „Wissensbestände“ zum Gegenstand der Reflexion gemacht werden müssten, die für bestimmte (Gruppen von) Schülerinnen und Schülern zu Barrieren werden können. Darüber hinaus erscheint es dringend geboten, die Auseinandersetzung mit Teilhabebarrieren im Schulsystem und den organisationalen Bedingungen und Möglichkeiten diskriminierungskritischen Handelns in allen Phasen der Lehrer(innen)bildung systematisch zu verankern.

Ein zweiter Aspekt der Professionalisierung betrifft die Notwendigkeit der Auseinandersetzung mit den biographischen Ressourcen der Schülerinnen und Schüler. Betrachtet man die biographischen Erzählungen unter dem Aspekt von Teilhabe- und Zugehörigkeitserfahrungen, kann man zusammenfassend feststellen, dass diese dann möglich werden, wenn die Subjekte sich mit ihren biographischen und sozio-kulturellen Ressourcen als „wirksam“ (Mecheril, 2003) erfahren können.

In der Erzählung von Anna Schuster gibt es eine Schlüsselerzählung, die aus der ansonsten als Leidensgeschichte präsentierten Hauptschulzeit heraussticht. Sie berichtet von Wirksamkeitserfahrungen, die sie im Unterricht beim Lesen und Vorlesen macht. Über ihre Erfolge im (Vor-)Lesen kann sie ihre schulischen Leistungsansprüche in den sprachlichen Fächern verbürgen und sich ein Mindestmaß an schulischer Anerkennung sichern. Das Lesen fungiert in ihrer Erzählung aber auch als eine Art Brücke, die es ermöglicht, Kontinuität zwischen verschiedenen Stationen ihrer Bildungsbiographie herzustellen. So konstruiert sie in ihrer Erzählung eine Verbindung zwischen der Bücherei in ihrem ländlichen Herkunftsort in Russland und der Schulbibliothek in der Hauptschule in Deutschland, die nach der Migration für sie zu einem signifikanten Ort wird, an dem ihre Liebe zu Büchern anschlussfähig wird. Angestoßen wird dies durch den Auftrag ihres Lehrers, im Deutsch-Förderunterricht die Bibliothek umzuräumen. Es lässt sich nicht mit Gewissheit sagen, ob dieser Aufgabe eine pädagogische Intention zugrunde liegt oder ob es sich eher um eine pragmatische „Beschäftigungsmaßnahme“ handelt. Unabhängig davon wird die Aufgabe für Anna Schuster jedoch zu einer entscheidenden Gelegenheitsstruktur, denn sie setzt die Aufgabe nicht nur rein technisch um, sondern beginnt die Bücher zu lesen und auszuleihen. 


\section{Teilhabechancen in der Migrationsgesellschaft |}

An dieser Geschichte wird zweierlei sichtbar: Erstens gibt es hier eine Möglichkeitsstruktur für etwas, das als biographische Zugehörigkeitsarbeit bezeichnet werden kann (vgl. Schwendowius, 2015, S. 499ff.). Darunter verstehe ich die „Arbeit" der Subjekte daran, biographische Ressourcen und Zukunftsentwürfe mit den Gelegenheitsstrukturen des jeweiligen (Bildungs-)Kontexts sinnhaft zu verknüpfen. Dies ermöglicht es den Einzelnen, sich in diesem Kontext als handlungsfähig und wirksam erfahren zu können. Voraussetzung dafür ist allerdings, dass diese Zugehörigkeitsarbeit (von den Institutionen und Professionellen) auch anerkannt wird.

Interessant ist zweitens der (vielleicht überraschende) Umstand, dass Zugehörigkeitsarbeit im vorliegenden Fall gerade über das Lesen möglich wird - einem Anforderungsbereich also, der in der Beschulung von Lernenden, die Deutsch als Zweitsprache erst erlernen müssen, oft eher als problematisch gilt. Im Fall „Anna Schuster" wird das Lesen aber - gewissermaßen erwartungswidrig - zu einer Ressource für Wirksamkeitserfahrungen und Schulerfolg. Dies lässt sich nur vor dem Hintergrund ihrer individuellen Bildungsbiographie verstehen, ihrer Lesesozialisation in Russland, der sie eine hohe biographische Relevanz verleiht und sie dadurch zu einer bildungsbiographischen Ressource macht. Daran wird auch deutlich, dass solche Verknüpfungsleistungen einer kreativen biographischen Eigenlogik folgen; sie können kaum prognostiziert oder gar durch standardisierte pädagogische Arrangements erzeugt werden.

Dennoch lässt sich daraus eine allgemeine Schlussfolgerung für die Lehrer(innen)bildung ziehen: Bedeutsam erscheint eine Sensibilisierung von Lehrkräften für die individuell-biographischen Ressourcen und Relevanzen der Lernenden. Diese Ressourcen ermöglichen zuweilen ganz unerwartete Verknüpfungen und Anschlüsse an schulische Erwartungsstrukturen. Indem sie es den Einzelnen ermöglichen, sinnhafte Verknüpfungen zwischen Biographie und Schule herzustellen, sind sie ein Potenzial für Wirksamkeitserfahrungen, das im schulischen Alltag aber meist unbeachtet bleibt. Für eine migrationsgesellschaftliche Öffnung von Schule könnte deshalb - über den Abbau struktureller Teilhabebarrieren hinaus die Frage interessant sein, wie die biographische Zugehörigkeitsarbeit von Schülerinnen und Schülern systematisch ermutigt, gestärkt und anerkannt werden kann.

\section{Anmerkungen}

1 Teile dieses Beitrags sind bereits in der zugrundeliegenden Dissertationsstudie (Schwendowius, 2015) publiziert worden.

2 Dies betrifft v. a. die 1990er Jahre. Inwiefern sich durch die faktische Abschaffung der Hauptschule als eigenständige Schulform in vielen Bundesländern sowie durch veränderte Modelle der DaZ-Förderung (vgl. Massumi \& von Dewitz 2015) neue Bedingungen ergeben und welche Folgen diese für die Empfehlungs- und Zuwei- 


\section{Teilhabechancen in der Migrationsgesellschaft}

sungspraxis am Übergang zur Sekundarschule haben, ist anderen Untersuchungen vorbehalten.

\section{Literatur}

Bade, K. J. \& Oltmer, J. (1999). Einführung: Aussiedlerzuwanderung und Aussiedlerintegration. Historische Entwicklung und aktuelle Probleme. In Dies. (Hrsg.), Aussiedler: deutsche Einwanderer aus Osteuropa (S. 9-51). Osnabrück: Universitätsverlag Rasch, IMIS-Schriften Band 8.

Baumert, J., Stanat, P. \& Watermann, R. (2006). Herkunftsbedingte Disparitäten im Bildungswesen. Differenzielle Bildungsprozesse und Probleme der Verteilungsgerechtigkeit. Wiesbaden: VS. http://dx.doi.org/10.1007/978-3-531-90082-7

Behrens, J. \& Rabe-Kleberg, U. (1992). Gatekeeping im Lebensverlauf - Wer wacht an Statuspassagen? Ein forschungspragmatischer Vorschlag, vier Typen von Gatekeeping aufeinander zu beziehen. In E. Hoerning (Hrsg.), Biographische Sozialisation (S. 101-136). Stuttgart: Lucius \& Lucius.

Bourdieu, P. \& Passeron, J. C. (1971). Die Illusion der Chancengleichheit. Stuttgart: Klett.

Bourdieu, P. \& Passeron, J. C. (2007). Die Erben. Studenten, Bildung und Kultur. Konstanz: UVK.

Bräu, K. \& Schlickum, C. (Hrsg.) (2015). Soziale Konstruktionen in Schule und Unterricht. Opladen: Budrich.

Budde, J. (2013). Einleitung. In: Ders. (Hrsg.). Unscharfe Einsätze: (Re-) Produktion von Heterogenität im schulischen Feld. Wiesbaden: Springer VS.

Dausien, B. (1996). Biographie und Geschlecht. Zur biographischen Konstruktion sozialer Wirklichkeit in Frauenlebensgeschichten. Bremen: Donat.

Dausien, B., Rothe, D. \& Schwendowius, D. (i. E.). Teilhabe und Ausgrenzung in Bildungsprozessen als biographische Erfahrung. In Dies. (Hrsg.), Bildungswege. Biographien zwischen Teilhabe und Ausgrenzung. Frankfurt: Campus.

Dravenau, D. \& Groh-Samberg, O. (2005). Bildungsbenachteiligung als Institutioneneffekt. Zur Verschränkung kultureller und institutioneller Diskriminierung. In P. A. Berger \& H. Kahlert (Hrsg.). Institutionalisierte Ungleichheiten. Wie das Bildungswesen Chancen blockiert (S. 103-129). München: Juventa.

Engelhardt, M. von (2011). Narration, Biographie, Identität. Möglichkeiten und Grenzen lebensgeschichtlichen Erzählens. In O. Hartung, I. Steininger \& T. Fuchs (Hrsg.), Lernen und Erzählen interdisziplinär (S. 39-60). Wiesbaden: VS.

Goffman, E. (1974). Stigma. Über Techniken der Bewältigung beschädigter Identität. Frankfurt: Suhrkamp. 


\section{Teilhabechancen in der Migrationsgesellschaft}

Gogolin, I. (2008). Der monolinguale Habitus der multilingualen Schule (2. Aufl.). Münster: Waxmann.

Gomolla, M. \& Radtke, F.-O. (2009). Institutionelle Diskriminierung. Die Herstellung ethnischer Differenz in der Schule (2. Aufl.). Wiesbaden: VS. http://dx.doi.org/10.1007/978-3-531-91577-7

Faulstich-Wieland, H., Weber, M. \& Willems, K. (2004). Doing Gender im heutigen Schulalltag. Empirische Studien zur sozialen Konstruktion von Geschlecht in schulischen Interaktionen. Weinheim: Juventa.

Hahn, A. (2000). Biographie und Lebenslauf. In Ders. (Hrsg.), Konstruktionen des Selbst, der Welt und der Geschichte. Aufsätze zur Kultursoziologie (S. 97-115). Frankfurt/M.: Suhrkamp.

Hoerning, E. (1989), Erfahrungen als biographische Ressourcen. In P. Alheit \& E. Hoerning (Hrsg.), Biographisches Wissen (S. 148-163). Frankfurt/M.: Campus.

Karakaşoğlu, Y., M. Gruhn, A. Wojcziechowicz (2011). Interkulturelle Schulentwicklung unter der Lupe. (Inter-)nationale Impulse und Herausforderungen für Steuerungsstrategien am Beispiel Bremen. Münster: Waxmann.

Kramer, R.-T. \& Helsper, W. (2010). Kulturelle Passung und Bildungsungleichheit - Potentiale einer an Bourdieu orientierten Analyse der Bildungsungleichheit. In H.-H. Krüger, U. Rabe-Kleberg, R.-T. Kramer \& J. Budde (Hrsg.). Bildungsungleichheit revisited. Bildung und soziale Ungleichheit vom Kindergarten bis zur Hochschule (S. 103-125). Wiesbaden: VS.

Massumi, M. \& von Dewitz, N. (2015). Neu zugewanderte Kinder und Jugendliche im deutschen Schulsystem. Bestandsaufnahme und Empfehlungen. Herausgegeben vom Mercator Institut und dem Zentrum für Lehrerbildung der Universität zu Köln.

Mecheril, P. (2003). Prekäre Verhältnisse. Über natio-ethno-kulturelle Zugehörigkeit. Münster: Waxmann.

Mecheril, P. (2004). Einführung in die Migrationspädagogik. Weinheim: Beltz.

Mecheril, P. \& Olalde, O.T. (2011). Die Religion der Anderen. Anmerkungen zu Subjektivierungspraxen der Gegenwart. In B. Aulenbach, U. Goel, M. Hummrich, C. Weissköppel (Hrsg.), Jugend, Migration und Religion. Interdisziplinäre Perspektiven (S. 35-66). Zürich: Nomos.

http://dx.doi.org/10.5771/9783845232362-33

Mecheril, P. \& Plößer, M. (2011). Differenzordnungen, Pädagogik und der Diversity-Ansatz. In R. Spannring, S. Arens, P. Mecheril et al. (Hrsg.), bildung macht - unterschiede. Facetten eines Zusammenhangs (S. 59-79). Innsbruck: Studia.

Rosenthal, G. (1995). Erlebte und erzählte Lebensgeschichte. Gestalt und Struktur biographischer Selbstbeschreibungen. Frankfurt/M.: Campus.

Said, E. W. (1978). Orientalism. New York. London: Routledge \& Kegan Paul. 


\section{Teilhabechancen in der Migrationsgesellschaft}

Schütze, F. (1987). Das narrative Interview in Interaktionsfeldstudien: erzähltheoretische Grundlagen. Teil I: Merkmale von Alltagserzählungen und was wir mit ihrer Hilfe erkennen können. Studienbrief der Fern-Universität Hagen.

Schwendowius, D. (2015). Bildung und Zugehörigkeit in der Migrationsgesellschaft. Biographien von Lehramts- und Pädagogikstudierenden. Bielefeld: transcript. http://dx.doi.org/10.14361/9783839431948

Terkessidis, M. (2010). Interkultur. Frankfurt/M.: Suhrkamp.

Wenning, N. (2007). Heterogenität als Dilemma für Bildungseinrichtungen. In S. Boller, E. Rosowski \& T. Stroot (Hrsg.), Heterogenität in Schule und Unterricht (S. 21-31). Weinheim: Beltz.

\section{Verfasserin}

Dr. ${ }^{\text {in }}$ Dorothee Schwendowius

Europa-Universität Flensburg

Auf dem Campus 1

D-24943 Flensburg

E-Mail: dorothee.schwendowius@uni-flensburg.de

Internet: www.uni-flensburg.de/erziehungswissenschaft/

wer-wir-sind/personen/schwendowius-dorothee-dr 